\title{
Effect of Calcium-Vitamin D Co-Supplementation on Insulin, Insulin Sensitivity, and Glycemia: A Systematic Review and Meta-Analysis of Randomized Clinical Trials
}

\author{
Authors \\ Omid Asbaghi, Mohammad Zeinali Khosroshahi, Sara Kashkooli, Amir Abbasnezhad
}

\author{
Affiliation \\ Nutritional Health Research Center, Lorestan University of \\ Medical Sciences, Khorramabad, Iran
}

Key words

vitamin D, calcium, glucose, insulin, systematic review, meta-analysis

$\begin{array}{lr}\text { received } & 17.01 .2019 \\ \text { accepted } & 27.03 .2019\end{array}$

Bibliography

DOI https://doi.org/10.1055/a-0887-0205

Horm Metab Res 2019; 51: 288-295

(c) Georg Thieme Verlag KG Stuttgart · New York

ISSN 0018-5043

Correspondence

Amir Abbasnezhad, PhD

Nutritional Health Research Center

Department of Nutrition

Lorestan University of Medical Sciences

Goledasht Blvd

P. O. Box 6813833946

Khorramabad

Iran

Tel.: + 98/912/8980 693, Fax: + 98/663/3300661

abbasnezhad.a@ajums.ac.ir
Supplementary Material for this article is available online at http://www.thieme-connect.de/products

\section{ABSTRACT}

We conducted a systematic review and meta-analysis of randomized controlled trials (RCTs) to assess the effect of calcium-vitamin D co-supplementation on insulin, insulin sensitivity, and glycemia. A systematic search was carried out in Web of Science, PubMed, EMBASE, Scopus, and Cochrane library without any language and time restriction up to 12 August 2018, to retrieve the RCTs, which examined the effect of calcium and vitamin D co-supplementation on fasting blood glucose (FBG), insulin, HOMA-B, HOMA-IR, and QUICKI. Meta-analyses were carried out using a random effects model, and $I 2$ indexes were used to evaluate the heterogeneity. Search yielded 2225 publications. Twelve RCTs with 4395 patients were eligible. Results demonstrated that calcium and vitamin D co-supplementation had significantly reducing effects on FBG, HOMA-IR and circulating levels of insulin. As the subgroup analysis demonstrated, shortterm ( $\leq 12$ weeks) calcium and vitamin D co-supplementation had a significant reducing effect on FBG. However, beneficial effects of calcium and vitamin D co-supplementation on circulating level of insulin and HOMA-IR were seen in both short-term and long-term (>12 weeks) supplementations. Furthermore, we found that high doses of vitamin $\mathrm{D}$ and calcium co-supplementation (vitamin $D \geq 2000 \mathrm{mg} /$ day and calcium $\geq 1000 \mathrm{mg} /$ day) had significantly reducing effects on FBG, HOMA-IR and insulin. Present meta-analysis indicated the beneficial effects of high-dose and short-term combined vitamin D and calcium supplementation on insulin, insulin resistance and glycemia; however, further large-scale RCTs with adequate and multiple dosing schedules are needed.

\section{Introduction}

Insulin resistance is present in most patients with glucose intolerance or non-insulin-dependent diabetes mellitus (NIDDM), and in $25 \%$ of normal-weight subjects with normal glucose tolerance [1]. In these situations, glucose intolerance can just be prevented when the $\beta$-cell is able to increase and maintain insulin secretion [1]. Failure to achieve this goal results in instability of glucose homeosta- sis, which is associated with many diseases [1]. An elevated blood level of glucose, insulin deficiency, and insulin resistance are the characteristic features of type 2 diabetes mellitus (DM) [2]. Type 2 DM has become a major problem for global health whose prevalence is expected to increase to 366 million by 2030 worldwide [3]. As one of the main causes of disability in 2015 [4], diabetes causes many social and economic problems and increases health costs 
worldwide [5]. Another complication associated with increasing insulin resistance and blood glucose levels is the cardiovascular disease, which requires intensive management of lipid and glucose concentration to reduce the complications [6]. The benefits of controlling serum levels of glucose in glucose related complications such as retinopathy, neuropathy and nephropathy have been reported in several randomized trials [7-9]. Although the treatment of these complications has improved over the past decades, the increasing prevalence of insulin resistance related complications highlight the importance of the innovative approaches for disease prevention and treatment.

There is a growing body of evidence suggesting that vitamin D status may influence several diseases, such as autoimmune disorders, cancer, diabetes, and cardiovascular disease [10]. The hypothesis of the possible association between diabetes and hypovitaminosis D was supported by detection of the nuclear receptor of vitamin D (VDR) in $\beta$-cells of the pancreas [11]. Polymorphism of VDR was associated with changes in insulin sensitivity and secretion, indicating vitamin D involvement in the pathogenesis of both types of diabetes [12,13]. Since then, numerous randomized clinical trials (RCTs) have been conducted to confirm or reject the beneficial effects of vitamin D supplementation in patients with diabetes, however, the results were controversial $[14,15]$. A systematic review and meta-analysis of the effect of vitamin D supplementation on glycemic control indicated that vitamin D supplementation had no significant effect on fasting blood glucose (FBG) [16].

Given the fact that vitamin $D$ and calcium can affect synergistically the function of pancreatic $\beta$-cells and improve the glycemic control [17], numerous studies have assessed the effect of calcium and vitamin D co-supplementation on glycemic control. A study by Pittas et al. [18] indicated that calcium and vitamin D co-supplementation in healthy adults with impaired fasting glucose (IFG) could attenuate increases in insulin resistance and glycemia. Whereas, another study by De Boer et al. [19] reported that calcium and vitamin D co-supplementation had no significant effect on parameters of glucose metabolism. In 2007, a systematic review and meta-analysis found that the combined calcium and vitamin D supplementation may be effective in preventing type 2 diabetes, especially in subjects with glucose intolerance [18]. Of note, they searched only MEDLINE through January 2007 and found only one RCTs regarding the calcium and vitamin D co-supplementation. Due to the inconsistent results found in the literature, we carried out a systematic review and meta-analysis of RCTs to assess the effect of calcium and vitamin D co-supplementation on insulin, insulin sensitivity, and glycemia.

\section{Materials and Methods}

We performed this systematic review and meta-analysis according to the guidelines of the 2009 preferred reporting items for systematic reviews and meta-analysis (PRISMA) statement [20].

\section{Search strategy}

In order to find articles related to the effect of vitamin D and calcium co-supplementation on glycemic indices, online databases Web of Science, PubMed, EMBASE, Scopus, and Cochrane library were systematically searched without any language and time restriction up to 12 August 2018, with the following search keywords in titles, abstracts and keywords: "vitamin D or cholecalciferol or ergocalciferol or alphacalcidol or alfacalcidol or calcitriol or paricalcitol or doxercalciferol or vitamin D2 or vitamin D3 “ and” diabetic or diabetes mellitus or glucose tolerance or insulin resistance or type 2 diabetes or FBG fasting blood glucose or HbA1c or hemoglobin A1c or HOMA-IR or homeostatic model assessment or Insulin or T2DM or fasting blood sugar or FBS “ and "Intervention or controlled trial or randomized or random or randomly or placebo or assignment or clinical trial or Trial”. We also used parentheses, asterisks and quotation marks to search for the exact terms and Boolean operators (AND and OR) for designing our search strategy. Moreover, a manual search and reference lists check of all included studies, and related reviews were performed to identify further relevant articles. Initially, 2225 studies were identified, of which finally 13 unique studies meeting inclusion criteria were selected for inclusion in this meta-analysis. The process of study selection is shown in the flow diagram ( $\triangleright$ Fig. 1). Reference management was carried out using EndNote X7.5. (Supplementary File 1, search strategy).

\section{Study selection}

Articles were eligible for inclusion in this meta-analysis if they fulfilled the following criteria:

1) RCTs of oral calcium and vitamin D co-supplementation; 2) trials with duration of more than one week; and 3) reported mean or median values in baseline and at the end of supplementation in intervention and control groups with SD, SEM or $95 \% \mathrm{CI}$. The exclusion criteria were as follows: 1) studies with no placebo group; 2) case-control, cross-sectional, cohort design and review studies; 3) studies which used a combination of other vitamins and minerals, including vitamin D and Calcium; 4) studies conducted in animal models; and 5) studies that were not available.

\section{Data extraction}

After removing duplicates, titles and abstracts of all studies were assessed independently by two reviewers (S.K. and M.Z.) to find potentially relevant studies for reading the full text. Finally, full text of each article was reviewed to determine whether the article is qualified for inclusion. Any disagreement about study inclusion was consulted with a third author (O.A.). Major demographic and clinical data of each included trial containing the following items of first author's name, publication year, and origin of country, study design, dosage and duration of Calcium and vitamin D co-supplementation and sample size in intervention and control groups were extracted. Demographic data included sex, mean age and body mass index (BMI), baseline and final values of metabolic variables (FBG, Insulin, HOMA-B, HOMA-IR, QUICKI) in control and treatment groups.

\section{Quality assessment}

Quality of each included study was assessed using 5-point scale Jadad score [21]. This scale assesses the quality of RCTs and includes random allocation (up to 2 points), double blinding (up to 2 points), and description of withdrawals and dropouts (up to 1 point). Studies with the scores of 3 and higher are generally considered as a high quality study. 


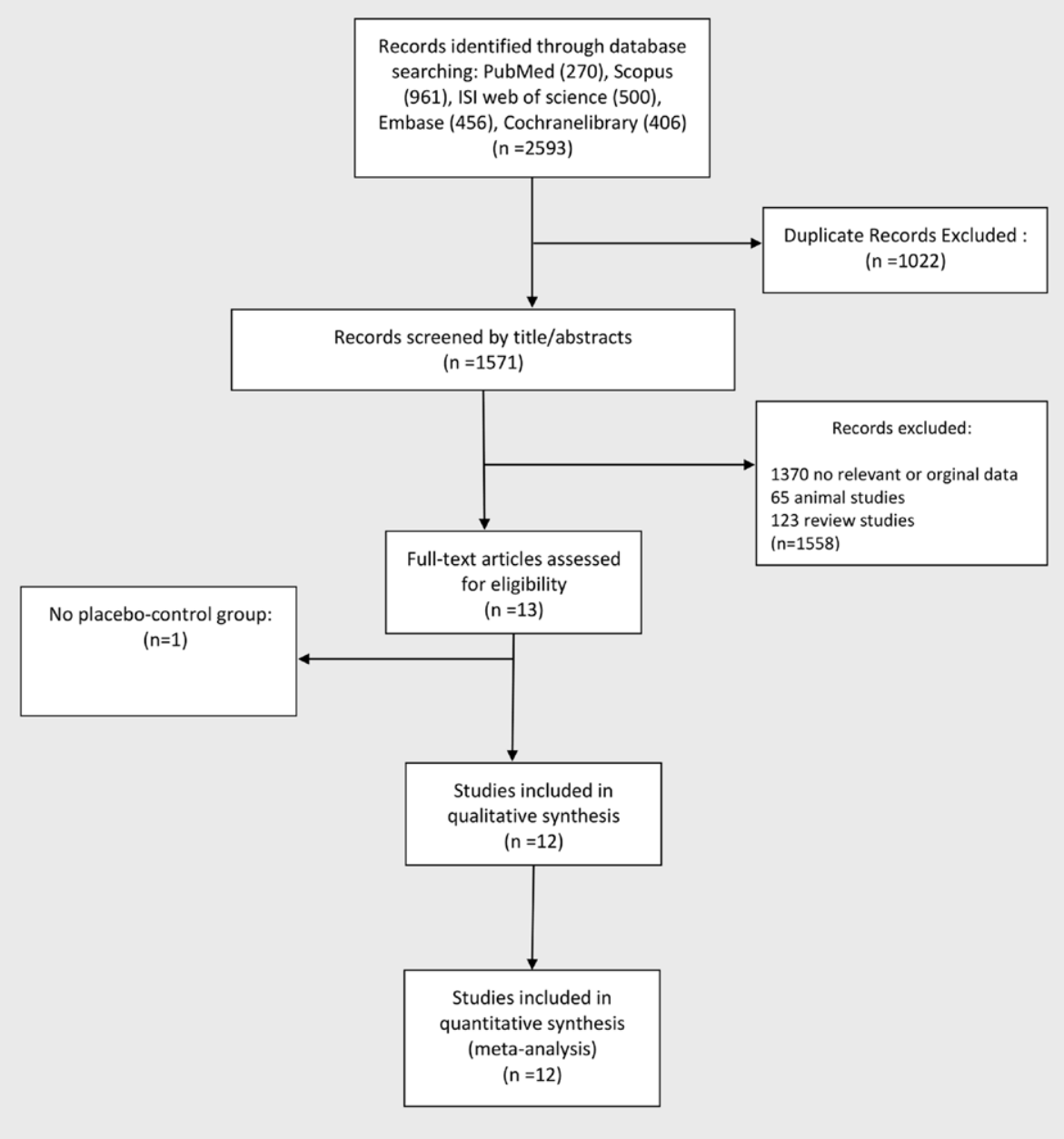

- Fig. 1 Flowchart of study selection for inclusion trials in the systematic review.

\section{Data synthesis and statistical analysis}

Mean and standard deviation (SD) of related variables at baseline and post intervention was used to compare changes between control and intervention groups. When S.D of change was not reported, it was calculated using following formula: s.d. = square root [(s.d. pre-intervention $)^{2}+(\text { s.d. post-intervention })^{2}-(2 \mathrm{R} \times$ s.d. pre-intervention $\times$ s.d. post-intervention)]. A correlation coefficient of 0.8 was assumed as R-value of the above-mentioned formula. Reported SE in some studies was converted to SD by multiplying $\mathrm{SE}$ with $\sqrt{\mathrm{n}}$, where $\mathrm{n}$ is the sample size of each group. If the variables were reported in median and range (or $95 \%$ confidence interval), mean and SD were estimated according to the method by Hozo et al., [22]. When the variables reported in the graphic form, the software GetData Graph Digitizer 2.24 [23] was used to digitize and extract the data. Random effects model was used to calculate pooled effect size of outcome data. We performed subgroup analysis based on pre-specific characteristics, including gender, health status, age, dose, study quality, study duration, and baseline levels of clinical parameters (HOMA-IR, FBG and Insulin) to detect possible sources of heterogeneity. Publication bias was assessed using visual assessment of funnel plots, Beg-test and Egger's regression asymmetry test. We performed the sensitivity analysis by conduct- ing one-study remove (leave-one-out) approach, to estimate the impact of each trial on the pooled effect size. Between-study heterogeneity was examined using Q test and I-square $\left(I^{2}\right)$ test [24]. All analyses were conducted using STATA v.12 (Stata Corporation, College Station, TX, USA).

\section{Results}

\section{Study selection}

In our initial search, a total of 2593 publications were found. Of these, 1022 duplicate articles were excluded initially, and remaining articles were assessed carefully by title and abstract. Upon screening the titles and abstracts, 13 full-text articles were retrieved and assessed for eligibility. Finally, 12 unique studies meeting inclusion and exclusion criteria were included in this meta-analysis. The process of study selection is shown in the flow diagram ( Fig. 1).

\section{Study characteristics}

All 12 studies [18, 19,25-34] were double-blinded and placebo-controlled studies. The intervention durations were from 6 to 312 weeks. 
Data are pooled from 12 studies with 4395 participants in the intervention arm and 4551 participants in the control arm. Age range of the participants was 18 to 71.7 years old. Seven studies were conducted in Iran [27-32, 34], 1 in Canada [25], and 4 in USA [18, 19, 26, 33]. All the included studies were parallel in design. Four studies were conducted on both genders [18, 26, 29, 33], and other studies enrolled females [19, 25, 27, 28, 30-32]. Basis on the jaded scale, 11 of the studies had good quality $(\geq 3)[18,19,25,26,28-34]$ and quality score of one study was lower than 3 [27]. The supplementation doses also varied between studies. Supplementation dose of calcium was ranged from $500 \mathrm{mg} /$ day to $1000 \mathrm{mg} /$ day, and for vitamin D ranged from $200 \mathrm{IU} /$ day to $50000 \mathrm{IU} /$ week. The supplementation was calcium citrate in one study [18], and in other studies, it was calcium carbonate [19, 25-34]. Some studies were conducted on healthy subjects $[18,19,25-27,32]$ and other studies were conducted on patients [28-31,33,34]. Characteristics of the included studies are summarized in $>$ Table 1.

\section{Publication bias}

Based on Egger's test, there was no statistical evidence of publication bias among the included studies in the overall analysis, except for effects of vitamin D and calcium co-supplementation on QUICKI $(p=0.028)$. (Supplementary File 1)

\section{Sensitivity analyses}

To evaluate the strength of our results, we made a sensitivity analysis. However, removing each individual study by sensitivity analysis did not change the pooled effect size of FBG, insulin, HOMA-B, HOMA-IR, and QUICKI.

\section{Meta-analysis}

The forest plots of the effects of vitamin D and calcium co-supplementation on glycemic status are shown in > Fig. 2 and supplementary ( $\triangleright$ Fig. 1S-4S). In this systematic review and meta-analysis, we pooled and analyzed data from 10 studies evaluating the effects of vitamin D and calcium co-supplementation on FBG, 6 studies on insulin, 5 studies on HOMA-B, 7 studies on HOMA-IR and 5 studies on QUICKI. Results indicated that vitamin D and calcium co-supplementation had significantly reducing effects on FBG (pooled WMD $=-2.28$ (95\% Cl: -3.76 to -0.80$), p=0.000$, $\mathrm{I}^{2}=74.3 \%$ ), insulin (pooled $\mathrm{WMD}=-0.85(95 \% \mathrm{Cl}:-1.60$ to -0.09$)$, $\mathrm{P}=0.000, \mathrm{I}^{2}=72.8 \%$ ), and also on HOMA-IR (pooled WMD $=-0.37$ (95\% Cl: -0.60 to -0.14$), p=0.000, \mathrm{I}^{2}=85.5 \%$ ). However, vitamin $\mathrm{D}$ and calcium co-supplementation had no significant effect on $\mathrm{HO}$ MA-B (pooled WMD $=-9.36$ (95\% Cl: -29.23 to 10.51, $\mathrm{p}=0.000$, $I^{2}=91.8 \%$ ) and QUICKI (pooled WMD $=0.01$ (95\% Cl: -0.02 to $\left.0.04), p=0.000, I^{2}=97.1 \%\right)$.

\section{Subgroup analyses}

\section{Effect of combined vitamin D and calcium} supplementation on FBG

Results of subgroup analyses ( $\triangleright$ Table $1 S$ ) indicated that heterogeneity disappeared in subgroups of subjects with baseline serum FBG $>100 \mathrm{mg} / \mathrm{dl}$, calcium $<1000 \mathrm{mg} /$ day or vitamin $\mathrm{D}<2000 \mathrm{IU} /$ day, participant's age $\geq 50$, and in healthy subjects. Co-supplementation of vitamin $D$ and calcium had significantly reducing effects on FBG in subgroups of subjects with baseline serum FBG $<100 \mathrm{mg} / \mathrm{dl}$, Trial's duration $\leq 12$ weeks, calcium doses $\geq 1000 \mathrm{mg} /$ day with vitamin D doses $\geq 2000 \mathrm{IU} /$ day, female participants, participant's age $<50$, and in high-quality studies with jadad score equal to 5 .

\section{Effect of combined vitamin D and calcium supplementation on insulin}

Results demonstrated that heterogeneity disappeared in subgroups of subjects with baseline serum insulin $<10 \mathrm{mg} / \mathrm{dl}$, Trial's duration $>12$ week, calcium $<1000 \mathrm{mg} /$ day or vitamin D $<2000 \mathrm{IU} /$ day, studies included both sexes, participant's age $\geq 50$, and in studies with the jaded score equal to 5 . Reducing effect of vitamin D and calcium co-supplementation on HOMA-IR was seen in all the subgroups except in subgroups of calcium doses $<1000 \mathrm{mg} /$ day or vitamin D doses $<2000$ IU/day, participant's age $\geq 50$, studies with the jadad scores lower than 5 and in healthy subjects ( $\triangleright$ Table $2 \mathbf{S}$ ).

\section{Effect of combined vitamin D and calcium supplementation on HOMA-IR}

Subgroup analyses indicated that heterogeneity disappeared in subgroups of subjects with baseline HOMA-IR $<1.4$, Trial's duration $>12$ weeks, calcium $<1000 \mathrm{mg} /$ day or vitamin $\mathrm{D}<2000 \mathrm{IU} /$ day, and high-quality studies with the jaded score equal to 5 ( $\triangleright$ Table $3 S$ ). Reducing effect of vitamin $D$ and calcium co-supplementation on $\mathrm{HO}$ MA-IR was seen in all the subgroups except in subgroups of calcium doses $<1000 \mathrm{mg} /$ day or vitamin D doses $<2000$ IU/day, participant's age $\geq 50$ and in healthy subjects.

\section{Discussion}

Results of the present meta-analysis demonstrated that calcium and vitamin $\mathrm{D}$ co-supplementation had significantly reducing effects on FBG, HOMA-IR, and circulating levels of insulin, whereas, this co-supplementation had no significant effects on HOMA-B and QUICKI. As the subgroup analysis demonstrated, short-term ( $\leq 12$ weeks) calcium and vitamin D co-supplementation had a significant reducing effect on FBG. However, beneficial effect of calcium and vitamin D co-supplementation on circulating level of insulin and HOMA-IR were seen in both short-term and long-term (> 12 weeks) supplementation. Furthermore, we found that high doses of vitamin $\mathrm{D}$ and calcium co-supplementation (vitamin $\mathrm{D} \geq$ $2000 \mathrm{mg} /$ day and calcium $\geq 1000 \mathrm{mg} /$ day) had significantly reducing effects on FBG, HOMA-IR, and insulin.

Several lines of evidence suggested the role of vitamin $D$ in the function of pancreatic $\beta$-cells. Vitamin $D$ seems to be able to improve insulin response to glucose [17]. Vitamin D may directly affect the function of $\beta$-cells through binding to the vitamin $D$ receptors in $\beta$-cells [35]. Recently, it has been shown that the enzyme activating vitamin D, 1 - $\alpha$-hydroxylase, is also expressed in $\beta$-cells [35]. Although, the beneficial effects of vitamin $D$ on the function of pancreatic $\beta$-cells are well documented, the results of the previous clinical trials regarding the effects of the vitamin D supplementation on glycemic control were inconsistent. A recent systematic review revealed that vitamin $D$ supplementation had no significant effect on FBG [16]. Moreover, a systematic review and meta-analysis found that there was no sufficient evidence for the effectiveness of vitamin D supplementation as a method to improve insulin resistance and normal FBG in type 2 diabetes patients [36]. A re- 


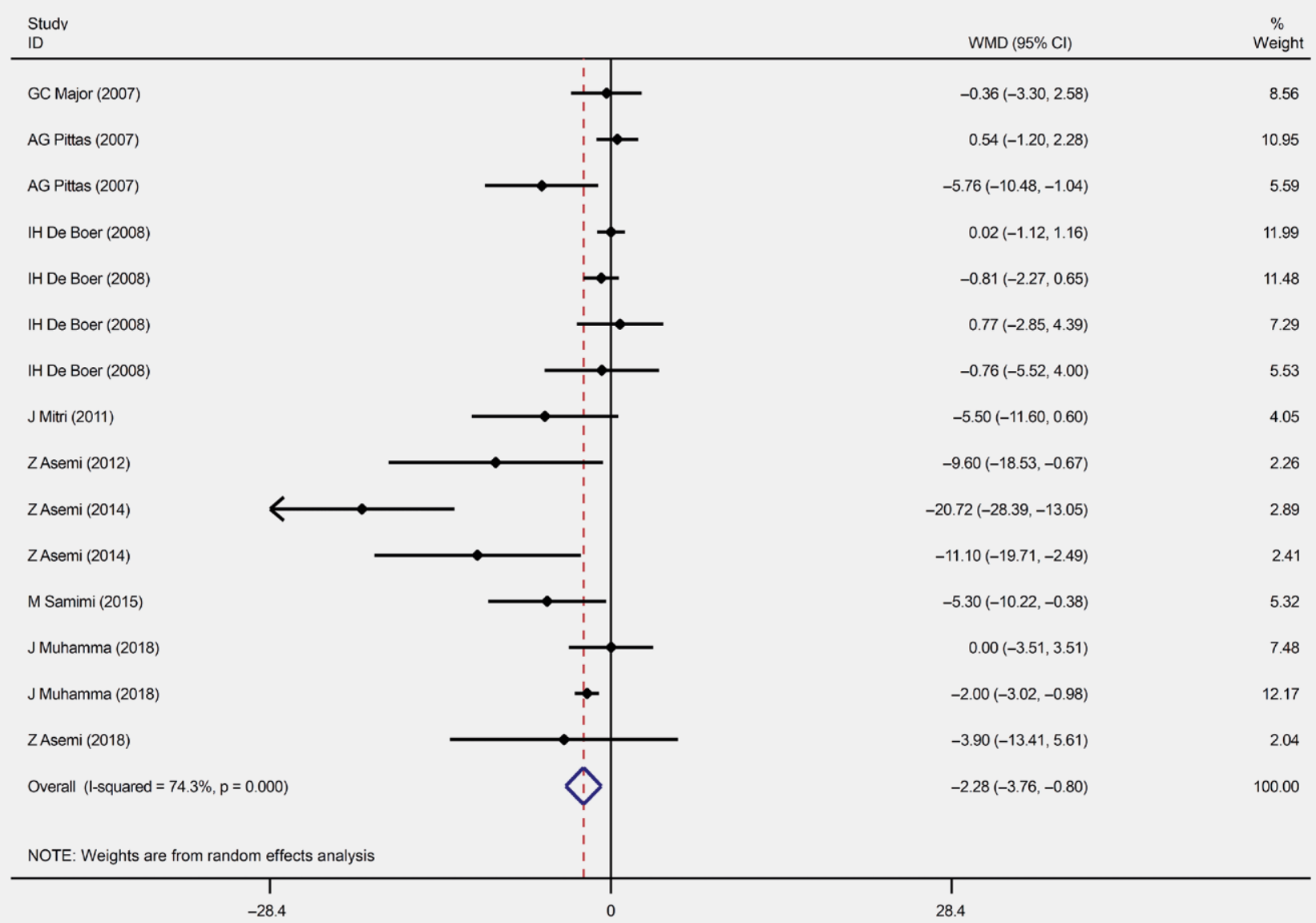

- Fig. 2 Forest plot of the random-effects meta-analysis of the effect of combined vitamin D and calcium supplementation on fasting blood glucose (FBG).

cent meta-analysis found that vitamin D supplementation had no significant reducing effect on FBG, however, subgroup analysis indicated that vitamin $D$ supplementation could improve glycemic control in vitamin D deficient type 2 DM patients [37].

Indirect effects of vitamin $D$ on the function of pancreatic $\beta$-cell may be mediated through its role in regulating calcium flux through the pancreatic $\beta$-cell [38]. The process of insulin secretion is highly dependent to calcium, thus, any changes in calcium flux can adversely affect the secretory function of $\beta$-cell [17]. In addition, a very narrow range of intracellular calcium is needed for insulin-dependent intracellular processes, therefore, any change of calcium levels in insulin-responsive tissues such as adipose tissue and skeletal muscles results in peripheral insulin resistance [38]. Although, basic and animal studies $[38,39]$ suggested that calcium and vitamin $D$ could synergistically improve the function of $\beta$-cells and glycemic control, results of the RCTs assessed the effect of vitamin D and calcium co-supplementation on insulin secretion and glycemic control were inconsistence. In 2007, a systematic review and meta-analysis reported that vitamin $\mathrm{D}$ alone had no effect in healthy individuals, but vitamin $\mathrm{D}$ and calcium co-supplementation may be beneficial in optimizing glucose metabolism in populations at risk for type 2 DM [17]. It should be noted that this systematic review and meta-analysis searched only MEDLINE for English-lan- guage literature through January 2007, and found only one study regarding vitamin $\mathrm{D}$ and calcium co-supplementation. Results of the present systematic review and meta-analysis indicated that calcium and vitamin D co-supplementation significantly reduced FBG, HOMA-IR, and insulin levels.

As subgroup analyses indicated, the beneficial effects of vitamin $D$ and calcium co-supplementation were seen in the subgroup of subjects with FBG levels lower than $100 \mathrm{mg} / \mathrm{dl}$, and not in subjects with FBG levels $\geq 100 \mathrm{mg} / \mathrm{dl}$. The possible reason that can be assumed for these results is that all the studies in the subgroup of $F B G \geq 100 \mathrm{mg} / \mathrm{dl}$ $[19,25,40]$ administered lower doses of vitamin D and calcium (vita$\min \mathrm{D} \leq 700 \mathrm{IU} /$ day and calcium $\leq 1000 \mathrm{mg} /$ day). In addition we found that co-supplementation of higher doses of calcium and vitamin D, (calcium $\geq 1000 \mathrm{mg} /$ day and vitamin $\mathrm{D} \geq 2000 \mathrm{IU} /$ day) had reducing effects on FBG, circulating levels of insulin and HOMA-IR. A growing body of evidence suggests that vitamin $D$ and calcium intakes higher than the current recommendations may lead to better health outcomes $[17,41]$. Results of the Nurses' Health Study indicated that a combined intake of $>1200 \mathrm{mg}$ calcium and $>800$ IU vitamin D was associated with a lower risk of type $2 \mathrm{DM}$ as compared with lower doses [42]. Optimal blood concentrations of $25(\mathrm{OH}) \mathrm{D}$ is critical for the positive effects of vitamin $D$ on glycemic control. Optimal blood concentrations of 25(OH)D for patients with DM has not been specified, but 
the most advantageous blood levels of $25(\mathrm{OH}) \mathrm{D}$ seems to be above $40 \mathrm{ng} / \mathrm{ml}$ [17]. Approximately $1000 \mathrm{IU} / \mathrm{d}$ of vitamin D are essential to achieve such a concentration [16]. In 2017, a systematic review and meta-analysis reported that the dose of vitamin D supplementation $\geq 1000 \mathrm{IU} /$ day had a reducing effect on FBG levels [37]. Recently, a systematic review and meta-analysis indicated that daily doses of vitamin D $\geq 2000$ IU/day had a positive impact on glycemic control [43]. In relation to calcium, it is difficult to determine the insufficiency of calcium status by biochemical methods, but suboptimal intake of calcium has been reported in different populations [44, 45]. Evidence suggests that daily intake of calcium more than $1200 \mathrm{mg}$ may be desirable for patients with DM [17].

In addition, the present meta-analysis indicated that short-term ( $\leq 12$ weeks) co-supplementation of calcium and vitamin D had a significant reducing effect on FBG. It should be noted that most short-term studies have used high doses of vitamin D and calcium for supplementation. Given the fact that achieving optimal circulating levels of $25(\mathrm{OH}) \mathrm{D}$ is critical for positive effects of vitamin $\mathrm{D}$ on glycemic control, thus, as described above, daily intake of highdose vitamin D is important to achieve this goal. Previous systematic review and meta-analysis studies, which examined the effect of vitamin D alone on glycemic control, have also reported that short-term supplementation of vitamin $D$ was beneficial in optimizing glucose metabolism. In 2015, a systematic review reported that short-term supplementation of vitamin D supplementation had a beneficial effect on glycemic control [16]. Furthermore, a recent systematic review and meta-analysis indicated that high-dose and short-term supplementation of vitamin $D$ alone had beneficial effects on insulin resistance and FBG [43]. However, in 2017, a systematic review and meta-analysis reported that neither short-term and nor long-term vitamin D supplementation has significant effect on FBG [46].

The present systematic review and meta-analysis have several strengths. We included RCTs which examined complementary endpoints, providing a comprehensive review on this topic. This review is based on an up to date literature search from a large number of databases and included 12 studies with 4395 participants in the intervention arm and 4551 participants in the control arm. Present study has also several limitations. First, we did not limit the systematic search to a particular disease, which led to an increase in heterogeneity. However, by subgroup analysis, heterogeneity decreased in some of the subgroups. Second, although we conducted a comprehensive search of the electronic literature, there might be studies that have not been included. Finally, the small sample size of the individual studies limits the strength of the conclusion of the present meta-analysis, however, we hope this study will be helpful for future studies.

\section{Conclusions}

In conclusion, present meta-analysis indicated that calcium and vitamin $D$ co-supplementation had reducing effects on FBG, HOMA-IR and circulating levels of insulin. However, the beneficial effects of combined vitamin $D$ and calcium supplementation were seen in higher doses of both calcium and vitamin D. Furthermore, present meta-analysis indicated that short-term co-supplementation of calcium and vitamin $\mathrm{D}$ can be effective in reducing FBS. How- ever, we found that both short-term and long-term co-supplementations has reducing effects on both HOMA-IR and circulating insulin. Further large-scale RCTs with adequate and multiple dosing schedules of calcium and vitamin D co-supplementation are needed to fully determine the role of calcium and vitamin D co-supplementation in optimizing glucose metabolism, especially in patients with diabetes.

\section{Author Contributions}

AA and OA designed the study. SK and MZK reviewed and selected the articles. OA and SK extracted needed data from articles. AA and OA performed data analysis and interpretation. AA drafted the manuscript.

\section{Funding}

This research did not receive any specific grant from funding agencies in the public, commercial, or not-for-profit sectors.

\section{Conflict of Interest}

The authors declare that they have no conflict of interest.

\section{References}

[1] Reaven GM. Role of insulin resistance in human disease (syndrome $X$ ): An expanded definition. Annu Rev Med 1993; 44: 121-131

[2] Narayan KM, Boyle JP, Geiss LS et al. Impact of recent increase in incidence on future diabetes burden: U.S., 2005-2050. Diabetes Care 2006; 29: 2114-2116

[3] Rathmann W, Giani G. Global prevalence of diabetes: Estimates for the year 2000 and projections for 2030. Diabetes Care 2004; 27: 2568-2569. author reply 2569

[4] [Anonymous]. Global, regional, and national incidence, prevalence, and years lived with disability for 310 diseases and injuries, 19902015: A systematic analysis for the Global Burden of Disease Study 2015. Lancet 2016; 388: 1545-1602

[5] Seuring T, Archangelidi O, Suhrcke M. The economic costs of type 2 diabetes: A global systematic review. PharmacoEconomics 2015; 33: 811-831

[6] Reaven GM. Pathophysiology of insulin resistance in human disease. Physiol Rev 1995; 75: 473-486

[7] [Anonymous]. Intensive blood-glucose control with sulphonylureas or insulin compared with conventional treatment and risk of complications in patients with type 2 diabetes (UKPDS 33). UK Prospective Diabetes Study (UKPDS) Group. Lancet 1998; 352: 837-853

[8] Kirkman MS, Mahmud H, Korytkowski MT. Intensive Blood glucose control and vascular outcomes in patients with type 2 diabetes mellitus. Endocrinol Metab Clin North Am 2018; 47: 81-96

[9] Akbari M, Ostadmohammadi V, Lankarani KB et al. The effects of alpha-lipoic acid supplementation on glucose control and lipid profiles among patients with metabolic diseases: A systematic review and meta-analysis of randomized controlled trials. Metabolism 2018; 87: 56-69

[10] Holick MF. The vitamin D deficiency pandemic: Approaches for diagnosis, treatment and prevention. Rev Endocr Metab Disord 2017; 18: $153-165$ 
[11] Chuang JC, Cha JY, Garmey JC et al. Research resource: nuclear hormone receptor expression in the endocrine pancreas. Mol Endocrinol 2008; 22: 2353-2363

[12] Zostautiene I, Jorde R, Schirmer $\mathrm{H}$ et al. Genetic variations in the vitamin $d$ receptor predict type 2 diabetes and myocardial infarction in a community-based population: The Tromso Study. PloS one 2015; 10 : e0145359

[13] Li L, Wu B, Liu JY et al. Vitamin D receptor gene polymorphisms and type 2 diabetes: A meta-analysis. Arch Med Res 2013; 44: 235-241

[14] Ryu $\mathrm{OH}$, Lee $\mathrm{S}$, Yu J et al. A prospective randomized controlled trial of the effects of vitamin D supplementation on long-term glycemic control in type 2 diabetes mellitus of Korea. Endocr J 2014; 61: 167-176

[15] Jehle S, Lardi A, Felix B et al. Effect of large doses of parenteral vitamin D on glycaemic control and calcium/phosphate metabolism in patients with stable type 2 diabetes mellitus: A randomised, placebo-controlled, prospective pilot study. Swiss Med Weekly 2014; 144: w13942

[16] Nigil Haroon N, Anton A, John J et al. Effect of vitamin D supplementation on glycemic control in patients with type 2 diabetes: A systematic review of interventional studies. J Diabetes Metab Disord 2015; 14: 3

[17] Pittas AG, Lau J, Hu FB et al. The role of vitamin D and calcium in type 2 diabetes. A systematic review and meta-analysis. J Clin Endocrinol Metab 2007; 92: 2017-2029

[18] Pittas AG, Harris SS, Stark PC et al. The effects of calcium and vitamin $D$ supplementation on blood glucose and markers of inflammation in non-diabetic adults. Diabetes Care 2007; 30: 980-986

[19] De Boer IH, Tinker LF, Connelly $S$ et al. Calcium plus vitamin D supplementation and the risk of incident diabetes mellitus in the Women's Health Initiative. Diabetes Care 2008; 31: 701-707

[20] Moher D, Liberati A, Tetzlaff ] et al. Preferred reporting items for systematic reviews and meta-analyses: The PRISMA statement. Ann Intern Med 2009; 151: 264-269

[21] Jadad AR, Moore RA, Carroll D et al. Assessing the quality of reports of randomized clinical trials: is blinding necessary? Control Clin Trials 1996; 17: 1-12

[22] Hozo SP, Djulbegovic B, Hozo I. Estimating the mean and variance from the median, range, and the size of a sample. BMC Med Res Methodol 2005; 5: 13

[23] Fedorov S. GetData graph digitizer. available at www.getdata-graph-digitizer.com 2008

[24] Higgins JP, Thompson SG, Deeks JJ et al. Measuring inconsistency in meta-analyses. BMJ 2003; 327: 557

[25] Major GC, Alarie F, Doré J et al. Supplementation with calcium + vitamin $D$ enhances the beneficial effect of weight loss on plasma lipid and lipoprotein concentrations-. Am J Clin Nutr 2007; 85: 54-59

[26] Mitri ], Dawson-Hughes B, Hu FB et al. Effects of vitamin D and calcium supplementation on pancreatic $\beta$ cell function, insulin sensitivity, and glycemia in adults at high risk of diabetes: The Calcium and Vitamin D for Diabetes Mellitus (CaDDM) randomized controlled trial. Am J Clin Nutr 2011; 94: 486-494

[27] Asemi Z, Tabassi Z, Heidarzadeh Z et al. Effect of calcium-vitamin D supplementation on metabolic profiles in pregnant women at risk for pre-eclampsia: a randomized placebo-controlled trial. Pak J Biol Sci 2012; 15: 316-324

[28] Asemi Z, Karamali M, Esmaillzadeh A. Effects of calcium-vitamin D co-supplementation on glycaemic control, inflammation and oxidative stress in gestational diabetes: A randomised placebo-controlled trial. Diabetologia 2014; 57: 1798-1806
[29] Tabesh M, Azadbakht L, Faghihimani E et al. Effects of calcium-vitamin $D$ co-supplementation on metabolic profiles in vitamin $D$ insufficient people with type 2 diabetes: A randomised controlled clinical trial. Diabetologia 2014; 57: 2038-2047

[30] Asemi Z, Foroozanfard F, Hashemi T et al. Calcium plus vitamin D supplementation affects glucose metabolism and lipid concentrations in overweight and obese vitamin $\mathrm{D}$ deficient women with polycystic ovary syndrome. Clin Nutr 2015; 34: 586-592

[31] Foroozanfard F, Jamilian M, Bahmani F et al. Calcium plus vitamin $D$ supplementation influences biomarkers of inflammation and oxidative stress in overweight and vitamin D-deficient women with polycystic ovary syndrome: a randomized double-blind placebo-controlled clinical trial. Clin Endocrinol 2015; 83: 888-894

[32] Asemi Z, Samimi M, Siavashani MA et al. Calcium-vitamin D co-supplementation affects metabolic profiles, but not pregnancy outcomes, in healthy pregnant women. Int J Prev Med 2016; 7: 49

[33] Muhammad J, Chan ES, Brown TT et al. Vitamin D supplementation does not affect metabolic changes seen with ART initiation. Open Forum Infect Dis 2017; 4: ofx210; doi: 10.1093/ofid/ofx210. eCollection 2017 Fall

[34] Samimi M, Kashi M, Foroozanfard F et al. The effects of vitamin D plus calcium supplementation on metabolic profiles, biomarkers of inflammation, oxidative stress and pregnancy outcomes in pregnant women at risk for pre-eclampsia. J Hum Nutr Diet 2016; 29: 505-515

[35] Sung CC, Liao MT, Lu KC et al. Role of vitamin D in insulin resistance. J Biomed Biotechnol 2012; 2012: 634195

[36] George PS, Pearson ER, Witham MD. Effect of vitamin D supplementation on glycaemic control and insulin resistance: A systematic review and meta-analysis. Diabet Med 2012; 29: e142-e150

[37] Wu C, Qiu S, Zhu X et al. Vitamin D supplementation and glycemic control in type 2 diabetes patients: A systematic review and meta-analysis. Metabolism 2017; 73: 67-76

[38] Harinarayan CV. Vitamin D and diabetes mellitus. Hormones (Athens, Greece) 2014; 13: 163-181

[39] Riachy R, Vandewalle B, Moerman E et al. 1,25-Dihydroxyvitamin D3 protects human pancreatic islets against cytokine-induced apoptosis via down-regulation of the Fas receptor. Apoptosis 2006; 11: 151-159

[40] Pittas AG, Harris SS, Stark PC et al. The effects of calcium and vitamin D supplementation on blood glucose and markers of inflammation in nondiabetic adults. Diabetes Care 2007; 30: 980-986

[41] Bischoff-Ferrari HA, Giovannucci E, Willett WC et al. Estimation of optimal serum concentrations of 25-hydroxyvitamin D for multiple health outcomes. Am J Clin Nutr 2006; 84: 18-28

[42] Pittas AG, Dawson-Hughes B, Li T et al. Vitamin D and calcium intake in relation to type 2 diabetes in women. Diabetes Care 2006; 29: 650-656

[43] Li X, Liu Y, Zheng Y et al. The effect of vitamin d supplementation on glycemic control in type 2 diabetes patients: a systematic review and meta-analysis. Nutrients 2018; 10: pii E375 doi:10.3390/nu10030375

[44] Fleming KH, Heimbach JT. Consumption of calcium in the U.S.: Food sources and intake levels. J Nutr 1994; 124: 1426s-1430s

[45] Lau EM, Suriwongpaisal P, Lee JK et al. Risk factors for hip fracture in Asian men and women: the Asian osteoporosis study. J Bone Miner Res 2001; 16: 572-580

[46] Lee C], lyer G, Liu Y et al. The effect of vitamin D supplementation on glucose metabolism in type 2 diabetes mellitus: A systematic review and meta-analysis of intervention studies. J Diabetes Complicat 2017; 31: $1115-1126$ 